\title{
Fabrication and characterization of a piezoresistive humidity sensor with a stress-free package
}

\author{
T. Waber ${ }^{1}$, M. Sax ${ }^{1}$, W. Pahl ${ }^{2}$, S. Stufler ${ }^{2}$, A. Leidl ${ }^{2}$, M. Günther ${ }^{3}$, and G. Feiertag ${ }^{1}$ \\ ${ }^{1}$ Munich University of Applied Sciences, Lothstraße 64, 80335 Munich, Germany \\ ${ }^{2}$ EPCOS AG, a member of TDK-EPC Corporation, Anzingerstraße 13, 81617 Munich, Germany \\ ${ }^{3}$ Technical University of Dresden, Helmholtzstraße 18, 01062 Dresden, Germany
}

Correspondence to: T. Waber (tobias.waber@hm.edu)

Received: 2 April 2014 - Revised: 7 July 2014 - Accepted: 9 July 2014 - Published: 8 August 2014

\begin{abstract}
A highly miniaturized piezoresistive humidity sensor has been developed. The starting point of the development was a $1 \times 1 \mathrm{~mm}^{2}$ piezoresistive pressure sensor chip. As sensing material, a polyimide was used that swells with increasing adsorption of water molecules. To convert the swelling into an electrical signal, a thin layer of the polyimide was deposited onto the bending plate of the pressure sensor. The humidity sensor was characterized in a climate chamber. The measurements show a sensitivity of $0.25 \mathrm{mV}$ per percent relative humidity (\%RH) and a non-linearity of 3.1\% full scale (FS) in the range of 30-80\% RH. A high cross-sensitivity to temperature of around $0.5 \mathrm{mV}^{\circ} \mathrm{C}^{-1}$ was measured, so temperature compensation is necessary. For stress-free packaging of the sensor chip, a novel packaging technology was developed.
\end{abstract}

\section{Introduction}

More and more sensors are integrated into mobile phones (see e.g. Lane et al., 2010). In the last four years this trend has accelerated. In 2010 no humidity sensors and almost no pressure sensors were integrated into mobile phones. Now, several mobile phones with humidity and pressure sensors are on the market, e.g. the Galaxy S4 phone from Samsung with the SHTC1 humidity sensor from Sensirion (Mayer and Lechner, 2013), or the Galaxy S3 with the LPS331AP pressure sensor from STMicroelectronics. The Bosch Sensortec company went one step further and developed a combined humidity, pressure and temperature sensor (BME280) with a size of $2.5 \times 2.5 \times 0.93 \mathrm{~mm}^{3}$ (Bosch, 2014). The main reason for combining pressure and humidity sensors into one package is that both need a cavity package with a port to the surrounding air. Besides the move towards combined pressure and humidity sensors, the trend towards smaller footprint and height will continue.

To fulfill these requirements, a piezoresistive humidity sensor with a chip size of only $1 \times 1 \mathrm{~mm}^{2}$ was developed. This sensor could be assembled together with a pressure sensor chip and an application-specific integrated circuit (ASIC) in a package with a size of $3 \times 2 \times 0.67 \mathrm{~mm}^{3}$.
The humidity of the air has a significant influence on our well-being. Therefore, a humidity sensor can provide important information for the user of a smart phone. Very dry air is a burden on our respiration system; high humidity could lead to the growth of mold. Besides that, the data provided by the humidity sensor are interesting for the manufacturers of smart phones. They can be used to exclude warranty in case of water damage, so the interest in a small digital humidity sensor in mobile phones or navigation systems, best packaged with a temperature and pressure sensor, is very high.

In this paper, we first introduce the state of the art in humidity sensing. Commercial humidity sensors are shown here and the difference compared to our sensor is explained. We will then show the design of the new sensor chip and will explain the manufacturing process. In Sect. 4, the static responses of the sensor to different values of the humidity and temperature are presented. For different accuracy requirements, possible calibration algorithms for temperature compensation are shown. Finally, a packaging solution is proposed. 


\section{State of the art in humidity sensing}

Several physical effects can be used to measure the humidity of the air. For capacity-type sensors, materials with a humidity-dependent dielectric constant are needed. Most polymers show this dependency. For impedance-type sensors, materials with a humidity-dependent resistance like salts or conductive polymers are used (Yamazoe and Shimizu, 1986; Sager et al., 1994). Also, resonant silicon cantilever sensors can detect the humidity. The resonance frequency of cantilevers decreases with increasing humidity due to the mass increase by adsorbed water molecules (Sone et al., 2004a, b; Wasisto et al., 2013). Furthermore, optical and gravimetric effects are used for humidity sensors (Lee and Lee, 2005).

Most commercial humidity sensors for mobile phones use the capacitive effect, e.g. the SHTC1 sensors from Sensirion, HTU21D from Measurement Specialties or Si7021 from Silicon Labs. The reasons for the choice of this working principle are cheap manufacturing, very low power consumption and a detection mechanism, which is very specific to humidity (Wagner et al., 2011; Lee and Lee, 2005).

An alternative to capacity-type sensors are piezoresistivetype sensors. Organic materials show a swelling when water from the surrounding air is absorbed. This effect is utilized in the hair tension hygrometer that was invented by de Saussure in 1783. The swelling can be converted into an electrical signal by making use of the piezoresistive effect. The first research work on piezoresistive humidity sensors was published by a group from the Technical University of Dresden (TUD) at the beginning of the 1990s (Sager et al., 1994; Gerlach and Sager, 1994; Buchhold et al., 1998a). A polymer layer on the top of a silicon-bending plate forms a bimorph that bends under the influence of humidity. Piezoresistors at the edge of the bending plate convert the mechanical stress caused by the humidity-induced bending into an electrical signal. Different polymer materials for piezoresistive humidity sensors were investigated at the Technical University of Dresden (Buchhold et al., 1998c; Buchhold et al., 1999; Guenther et al., 2001). However, this work did not result in a commercial sensor, and as far as we know, no further investigations for commercial piezoresistive humidity sensors were made. For use in mobile phones or navigation systems, the sensors have to be smaller, cheaper and suitable for production in bulk. Based on the prior work, we built a very small humidity sensor chip with a chip size of only $1 \times 1 \mathrm{~mm}^{2}$. Compared to prior work, the chip area was reduced by a factor of 10 , while still maintaining excellent electrical performance. A challenge for the fabrication of piezoresistive humidity sensors is the stress-free packaging. Based on experiences with the packaging of piezoresistive pressure sensors, this problem was solved by developing a technology where the sensor chip is flip-chip bonded onto electroplated copper springs (Waber et al., 2013). The humidity sensor chip is compatible with this package.

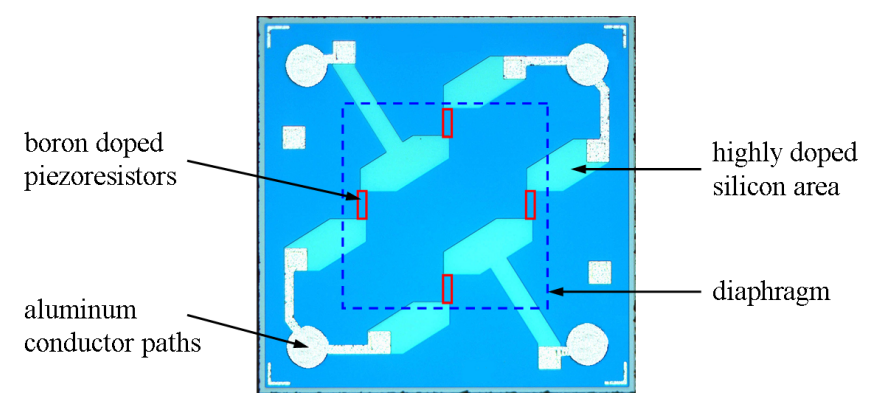

Figure 1. Pressure sensor chip with four piezoresistors interconnected as a Wheatstone bridge. The doping of the piezoresistors is too low to be visible in an optical microscope image. The location of the resistors is therefore indicated by red boxes. The footprint is $1 \times 1 \mathrm{~mm}^{2}$ and the height $150 \mu \mathrm{m}$.

\section{Design of the humidity sensor chip}

A piezoresistive pressure sensor chip, which will be presented in Sect. 3.1, was the starting point of this work. In order to develop a humidity sensor, a thin layer of the polyimide was deposited onto the bending plate of the pressure sensor chip and was used as a sorption-mechanical transducer. Combining a polymer and a micro-fabricated pressure sensor chip made it possible to monitor the humiditydependent swelling of a polymer continuously. The material properties, the layout and the processing of the polymer layer will be shown in Sect. 3.2. Calculations of the sensitivity and the noise of the humidity sensor are shown in Sect. 3.3.

\subsection{Piezoresistive pressure sensor}

Figure 1 shows the top view of the pressure sensor chip. This chip is applied as the sensing element of the T5400 pressure sensor module from EPCOS (Waber et al., 2013). The chip was fabricated using silicon on insulator (SOI) technology (see Fig. 2). First, cavities were etched into a silicon wafer by $\mathrm{KOH}$ etching (Fig. 2, step 2). A second silicon wafer was then bonded onto the cavity wafer (Fig. 2, step 3). The diaphragms (bending plates) were formed by grinding the second wafer down to a thickness of $10 \mu \mathrm{m}$ (Fig. 2, step 4). On the bending plates, four piezoresistors were formed by doping. To achieve a high sensitivity, the piezoresistors were placed at the edge of the diaphragm, where the stress is at its maximum (Fig. 2, step 5). The resistors were connected as a Wheatstone bridge by highly doped areas (Fig. 2, step 6) and aluminum lines (Fig. 2, step 7). The sensor gives an output voltage that is proportional to the surrounding air pressure.

\subsection{Polymer layer}

Polymer layers absorb water molecules from the surrounding air. The absorption of water results in a swelling of the material. In layers with a thickness of a few $\mu \mathrm{m}$, an equilibrium between the humidity of the surrounding air and the humidity 
(1)

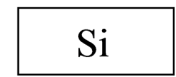

(2)

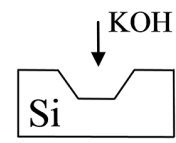

(3)

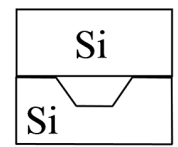

(4)

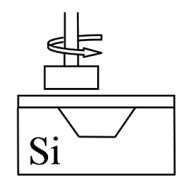

(5)

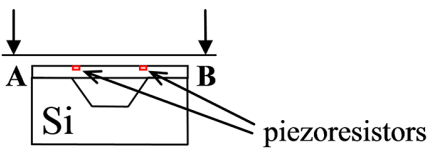

(6)

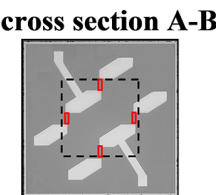

(7)

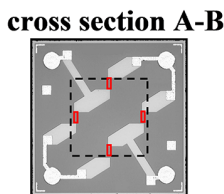

Figure 2. Pressure sensor chip fabricated in SOI technology. (1) A pure silicon wafer. (2) Cavities are etched by $\mathrm{KOH}$ etching. (3) A second silicon wafer is bonded onto the cavity wafer. (4) The bending plates are formed by grinding the second wafer. (5) Four piezoresistors are formed by doping on the bending plate. (6) The resistors are connected as a Wheatstone bridge by highly doped areas and (7) aluminum lines.

in the polymer is reached within minutes. It was shown earlier that the swelling is completely reversible (Buchhold et al., 1999), so thin polymer layers can be used to convert the relative humidity of the air into a swelling. The sensor incorporates the following chain of transducers:

- The polymer layer converts the humidity in the surrounding air into an in-plane expansion (sorptionmechanical transducer).

- Polymer layer and silicon-bending plate form a bimorph that transforms the expansion into a bending (mechanical transducer).

- The deformation of the bending plate results in a change in the stress in the piezoresistors.

- The piezoresistors transform the stress into a change in electrical resistance (mechano-electrical transducer).

As a humidity-responsive polymer material, the photosensitive Polyimide Durimide 7505, obtained from Fujifilm Electronic Materials Co., was used in the present work. In older publications the trade name Probimide is used for the same material. In the humidity sensor the Durimide 7505 layer has a thickness of $3 \mu \mathrm{m}$. This thickness was chosen as a compromise between sensitivity and response time. A higher sensitivity could be reached by using a thicker polyimide layer. Unfortunately, the response time also increases with increasing thickness because more time is needed for the diffusion of the water molecules into the polymer layer (Buchhold et al., 1998d).

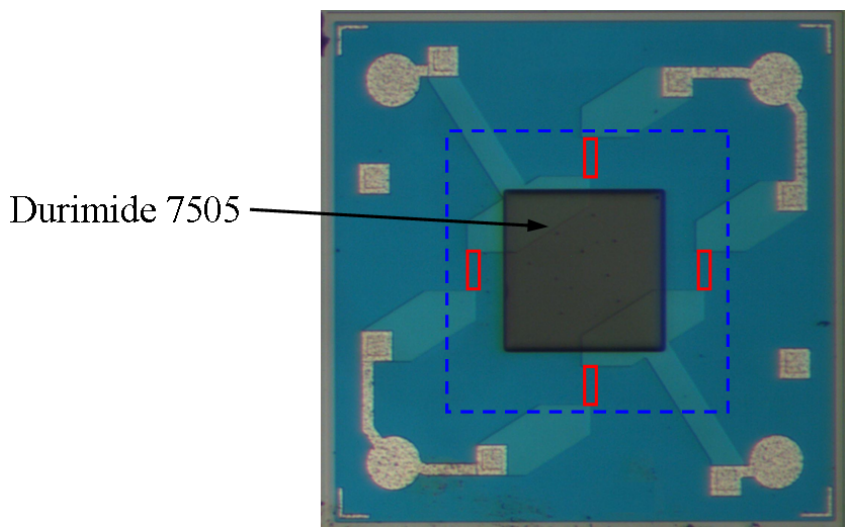

Figure 3. Sensor chip with a Durimide 7505 layer on top of the diaphragm. The patch has a size of $300 \times 300 \mu \mathrm{m}$ and a thickness of $3 \mu \mathrm{m}$.

Patches with a size of $300 \times 300 \mu \mathrm{m}^{2}$ were patterned in the center of the bending plate using a photolithographic process (see Fig. 3). To avoid cross-sensitivity to the air pressure, a port was etched in the bottom of the chip by deep reactive ion etching (DRIE) in order to open the reference volume (see Fig. 4).

For the characterization of the Durimide 7505, the absorption of water was first calculated and then measured.

The moisture loading capacity was characterized by a relation of the mass of the absorbed water to the mass of the dry polymer using the value of a saturation concentration $c_{\mathrm{s}}$ given by (Buchhold et al., 1998a)

$$
\begin{aligned}
c_{\mathrm{S}}(\varphi, T) & =\frac{m_{\text {water }}(\varphi, T)}{m_{\text {dry polymer }}}= \\
& \frac{m_{\text {coated wafer }}(\varphi, T)-m_{\text {coated wafer }}(0 \% \mathrm{rh})}{m_{\text {coated wafer }}(0 \% \mathrm{rh})-m_{\text {uncoated wafer }}} .
\end{aligned}
$$

At $100 \% \mathrm{RH}$, the saturation concentration reaches its $\operatorname{maximum} c_{\mathrm{s}, \max }$.

\subsubsection{Calculation}

The datasheet value of the saturation concentration $c_{\mathrm{S}}$ of Durimide 7505 at $50 \%$ RH and room temperature is $1.08 \%$. Assuming a linear relation between humidity and moisture absorption, the maximum saturation concentration $c_{\mathrm{s}, \max }$ is $2.16 \%$.

\subsubsection{Measurement}

For the measurement, first, an uncoated silicon wafer was weighed using a precision balance Sartorius BP210D. The wafer was then coated with a $3 \mu \mathrm{m}$ thick polyimide layer and was weighed in a chamber at defined values of temperature $T$ and relative humidity $\varphi$ of the surrounding air $(T=19$ $\left.25^{\circ} \mathrm{C}, \varphi=0-85 \% \mathrm{RH}\right)$. Ambient conditions were controlled 


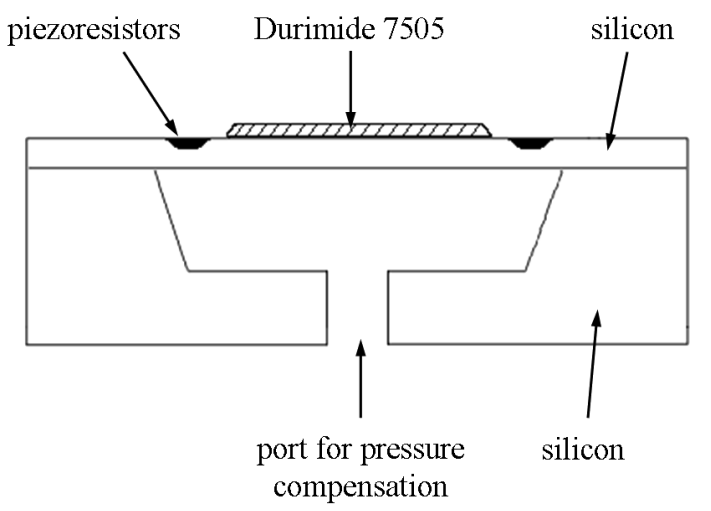

Figure 4. Schematic cross section of the sensor chip with a Durimide layer (not to scale). For pressure compensation, a port was etched onto the bottom of the chip.

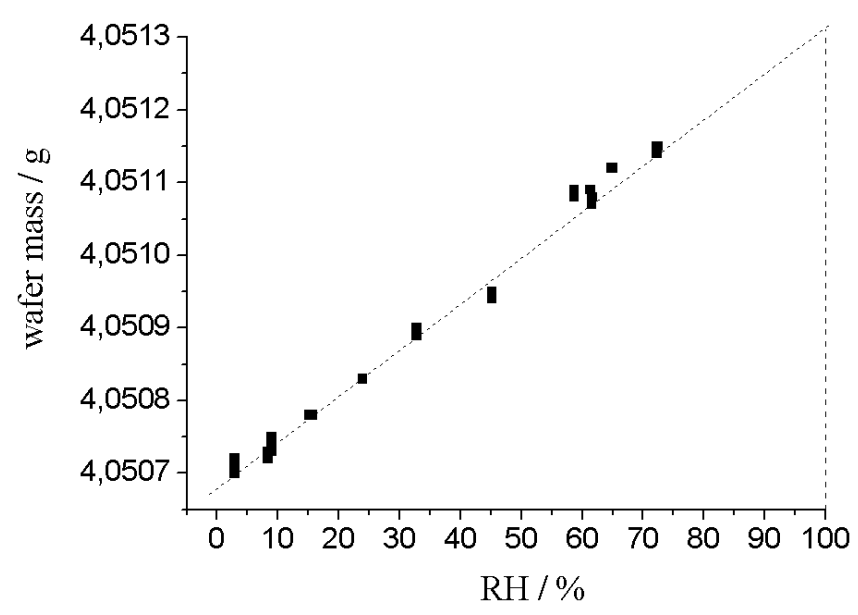

Figure 5. Relation between relative humidity and wafer mass. At $100 \% \mathrm{RH}$, the wafer mass and the saturation concentration $c_{\mathrm{S}}$ (Eq. 1) reach the maximum. By averaging the results from four wafers, we found that the maximum loading capacity $c_{\mathrm{s}, \max }$ of Durimide 7505 was $3.2 \%$.

with a Vaisala HMP 230 humidity and temperature sensor with uncertainties of $\pm 0.5 \mathrm{~K}$ and $\pm 2 \% \mathrm{RH}$, respectively. As a result, a linear relation between the relative humidity and the wafer mass was found, as shown in Fig. 5. By averaging the results from four wafers, we found that the maximum loading capacity of Durimide 7505 was $3.2 \%$.

The disagreement between the datasheet value and the measurements could be explained by different curing conditions of the polyimide layers. In Table 1, the measured result is compared with the loading capacity of other polymers that were investigated earlier by the TU Dresden in the 90s (Buchhold et al., 1998b). It can be seen that the maximum saturation concentration is comparable to other polymers used for humidity sensors. For humidity sensors, a high maximum loading capacity is desired, because it results in a high expansion and a high sensitivity.
Table 1. Maximum loading capacity of Durimide 7505 and the polymers measured in the reference (Buchhold et al., 1998b).

\begin{tabular}{lr}
\hline Polymer & $c_{\mathrm{s}, \max , \text { lin }} / \%$ \\
\hline PI2525 & 3.4 \\
PI2560 & 3.3 \\
PI2566 & 1.9 \\
PI2611 & 2.0 \\
Photo-BCB & 0.3 \\
Durimide 7505 & 3.2 \\
\hline
\end{tabular}

\section{Characterization of the piezoresistive humidity sensor}

The response of the sensor to humidity and temperature was investigated. In the following sections, first the sensitivity and noise of the humidity sensor is calculated, and then the set-up and the results of the measurements are shown.

\subsection{Calculations of sensitivity and noise}

\subsubsection{Calculation of the sensitivity}

An equation for the calculation of the bridge voltage of a piezoresistive pressure sensor was given by Gerlach and Sager (Gerlach and Sager, 1994):

$U_{\text {out }}(\varphi)=U_{0} \cdot \frac{3}{2}\left(\pi_{\mathrm{L}}-\pi_{\mathrm{T}}\right) \cdot \frac{E_{\mathrm{PI}}}{1-v_{\mathrm{Si}}} \cdot \frac{h_{2}}{h_{1}} \cdot \frac{b_{1}}{b_{1}+b_{2}} \cdot \alpha_{l, \varphi} \cdot \varphi$.

Here $U_{0}$ is the supply voltage; $\pi_{\mathrm{L}}, \pi_{\mathrm{T}}$ the piezoresistive coefficients in the longitudinal and transversal directions; $E_{\mathrm{PI}}$ the Young modulus of polyimide; $\nu_{\mathrm{Si}}$ the Poisson ratio of silicon; $h_{1}$ the thickness of the membrane and $h_{2}$ the thickness of polyimide; $b_{1}$ the width of the coated membrane and $b_{2}$ the width of the uncoated membrane; $\alpha_{1, \varphi}$ the humidity-induced length expansion coefficient; $\varphi$ the relative humidity.

The derivative of the output voltage $U_{\text {out }}$ of the humidity $\varphi$ is the sensitivity Sens (see Eq. 3).

$$
\begin{aligned}
& \text { Sens }=\frac{\mathrm{d} U_{\text {out }}}{\mathrm{d} \varphi}= \\
& U_{0} \cdot \frac{3}{2}\left(\pi_{\mathrm{L}}-\pi_{\mathrm{T}}\right) \cdot \frac{E_{\mathrm{PI}}}{1-\nu_{\mathrm{Si}}} \cdot \frac{h_{2}}{h_{1}} \cdot \frac{b_{1}}{b_{1}+b_{2}} \cdot \alpha_{1, \varphi} .
\end{aligned}
$$

With the coefficient of humidity expansion $\alpha_{l, \varphi}=32 \times$ $10^{-6} / \% \mathrm{RH}$, determined by Buchold (Buchhold et al., 1998b), the geometry parameters of the sensor chip and a supply voltage, $U_{0}=3 \mathrm{~V}$, we obtain for the sensitivity

Sens $=0.15 \frac{\mathrm{mV}}{\% \mathrm{RH}}$.

\subsubsection{Estimation of the noise}

The EPCOS pressure sensor chip has a sensitivity of $0.018 \mathrm{mV} / \mathrm{V} / \mathrm{hPa}$. For a supply voltage of $3 \mathrm{~V}$, the sensor has 


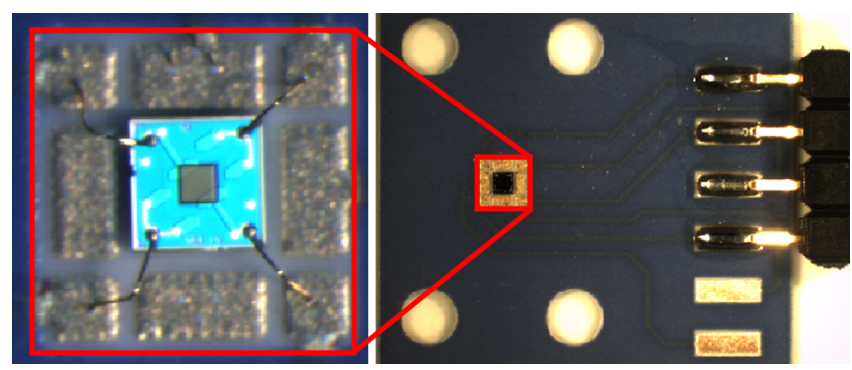

Figure 6. Humidity sensor chip is wire bonded onto a ceramic substrate. To avoid external mechanical stress, the sensor is not glued to the substrate. Mechanical stress would influence the measurement results.

a sensitivity of $0.054 \mathrm{mVhPa}^{-1}$. The noise of the pressure sensor with ASIC is about $0.03 \mathrm{hPa}$, so the noise of the humidity sensors can be estimated as follows:

$\mathrm{RH}_{\text {noise }}=\frac{0.054 \frac{\mathrm{mV}}{\mathrm{hPa}}}{0.15 \frac{\mathrm{mV}}{\% \mathrm{RH}}} \cdot 0.03 \mathrm{hPa}=0.011 \% \mathrm{RH}$.

The uncertainty of the pressure sensor caused by external stress or thermally induced stress is less than $1 \mathrm{hPa}$ (Waber et al., 2014). By converting this into humidity, an uncertainty of $<0.4 \%$ RH can be estimated. This uncertainty and the noise are far below the typical accuracy requirements of around $3 \% \mathrm{RH}$. In order to achieve short response times, the thickness of the polymer could be reduced, with only moderate impact on the accuracy.

\subsection{Measurement set-up}

The characterization of the sensor chip was carried out with a semi-automatic measurement set-up. The main components are a WEISS WKL 64 climate chamber and a Keithley 3706 system switch/multimeter with an extension multiplexer card. As reference sensors, a capacitive humidity sensor from the $\mathrm{E}+\mathrm{E}$ company (accuracy at $20^{\circ} \mathrm{C}: \pm 2 \% \mathrm{RH}$ $(0 \ldots 90 \% \mathrm{RH}))$ and a PT100 temperature sensor were used. As shown in Fig. 6, the sensor chips were connected to ceramic substrates by wire bonds. To avoid packaging stress, the sensors were not glued to the substrate. Only the bonding wires hold the chip in place. A die bonding material between the chip and the substrate would cause a mechanical stress, which would influence the measurement results.

\subsection{Measurement results of the humidity sensor}

In the next two sections, results of the measurements are presented. First, the response to humidity at constant temperature was investigated. The response to temperature at constant humidity was then measured.

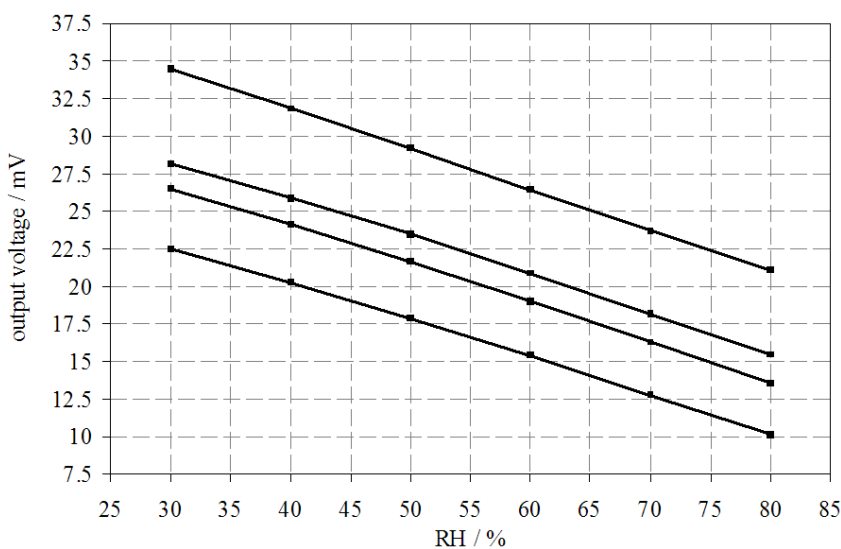

Figure 7. Relation between relative humidity and output voltage for four sensors. The sensitivity of the four sensors is between $0.24 \mathrm{mV} / \% \mathrm{RH}$ and $0.26 \mathrm{mV} / \% \mathrm{RH}$. The fact that all four curves are almost parallel is interesting for a one-point calibration (see Sect. 4.4).

\subsubsection{Response to humidity}

The measurement set-up described above was used. The supply voltage of the Wheatstone bridge was set to $3 \mathrm{~V}$. The temperature was kept constant at $25^{\circ} \mathrm{C}$. The output bridge voltage of four different sensors was measured at different values of the relative humidity in the range from 30 to $80 \% \mathrm{RH}$. The results are shown in Fig. 7. The output voltage is inversely proportional to the relative humidity. The output spans are around $12.5 \mathrm{mV}$ for the humidity range from 30 to $80 \% \mathrm{RH}$. The different offsets are mainly caused by manufacturing variations of the mechano-electrical transducer. Different resistances between aluminum and the highly doped silicon cause the variations.

For the four humidity sensor chips, the mean sensitivity was determined to be $0.25 \mathrm{mV} / \% \mathrm{RH}$. This result agrees quite well with the value of $0.15 \mathrm{mV} / \% \mathrm{RH}$ that was calculated in Sect. 4.1. All four sensors showed nearly similar sensitivities, so a one-point calibration could be sufficient for many applications. Possible calibration procedures are shown in Sect. 4.4.

The measured sensitivity is three times higher than the sensitivities obtained at the TU Dresden in the 1990s (Gerlach and Sager, 1994).

\section{Linearity}

The linearity between humidity and output voltage was investigated. Figure 8 shows the measurement results at different values of the relative humidity as well as a linear interpolation between the points at 30 and $80 \% \mathrm{RH}$. The linearization error reaches a maximum at $55 \% \mathrm{RH}$. For the four sensors, the linearization error was found to be in the range from 0.5 to $3.1 \%$ full scale (FS). Prior results by the TU Dresden were also in the range from 0.5 to $5 \%$ FS for 


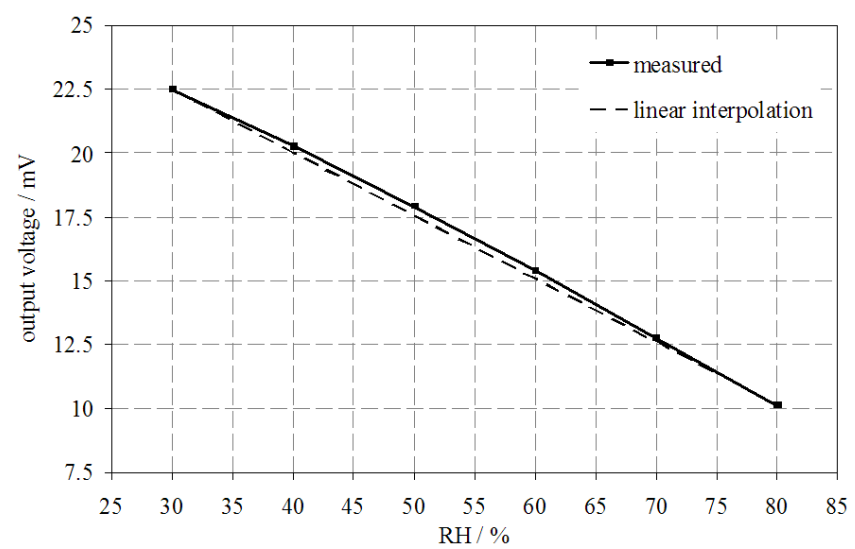

Figure 8. Comparison of the output voltage measured with the linear interpolation of the endpoints at 30 and $80 \% \mathrm{RH}$. The maximum error for four sensors was $3.1 \% \mathrm{FS}$, so linear interpolation is not sufficient if an accuracy of better than $3 \% \mathrm{FS}$ is required.

a humidity range from 10 to $95 \%$ RH (Gerlach and Sager, 1994). The error caused by the non-linearity can be reduced significantly by a quadratic interpolation. If the accuracy requirement is high $(<3 \% \mathrm{FS})$, a third measurement point at $55 \% \mathrm{RH}$ would be necessary for the calibration of the sensor.

\section{Response time}

To compare the dynamic behavior of our humidity sensor chip with the Sensirion SHT25 humidity sensor, a step response was measured at $25^{\circ} \mathrm{C}$ for both. The time to reach $63 \%$ of the saturation output voltage was $21 \mathrm{~s}$ for our sensor and $14 \mathrm{~s}$ for the SHT25. However, the polyimide layer is not optimized yet. The response time could be reduced by decreasing the thickness of the polyimide. Decreasing the thickness would also lead to an uncritical reduction of the sensitivity.

\subsubsection{Cross-sensitivity to temperature}

Piezoresistive sensors show a strong cross-sensitivity to temperature. At a constant humidity of $30 \% \mathrm{RH}$, the output bridge voltage of the sensors was measured at temperatures from 25 to $40^{\circ} \mathrm{C}$. The results are shown in Fig. 9. A temperature change of $5^{\circ} \mathrm{C}$ alters the output voltage by $2.5 \mathrm{mV}$. If this value is compared with the humidity sensitivity of $0.25 \mathrm{mV} / \% \mathrm{RH}$, it becomes clear that temperature compensation is necessary for any practical use of the sensor. As this is also standard for piezoresistive pressure sensors, it is not an obstacle to an application of the sensor. Temperature sensors can easily be integrated into the ASIC of the sensor module. In Sect. 4.4, it is shown how cross-sensitivity to temperature can be compensated for.

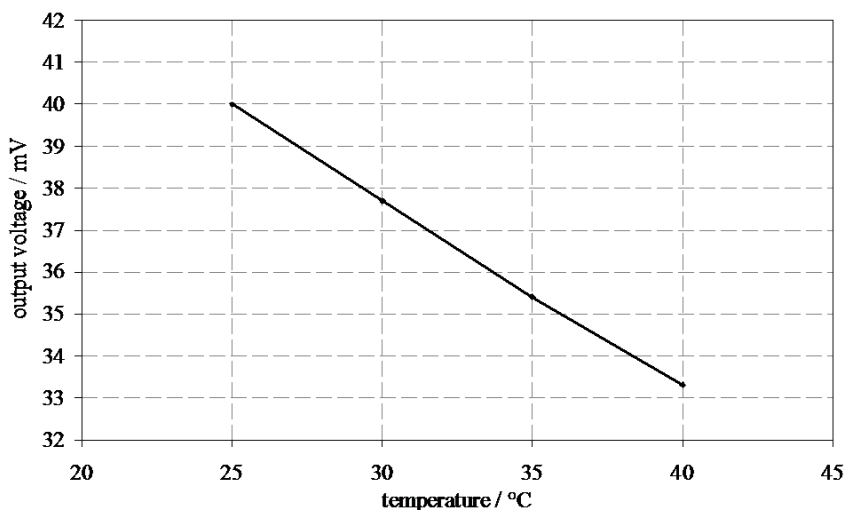

Figure 9. Bridge output voltage of the sensor at the relative humidity of $30 \% \mathrm{RH}$ and at different temperatures. As can be seen, the sensor has a strong cross-sensitivity to temperature. By changing the temperature by $5^{\circ} \mathrm{C}$, the output voltage alters by around $2.5 \mathrm{mV}$.

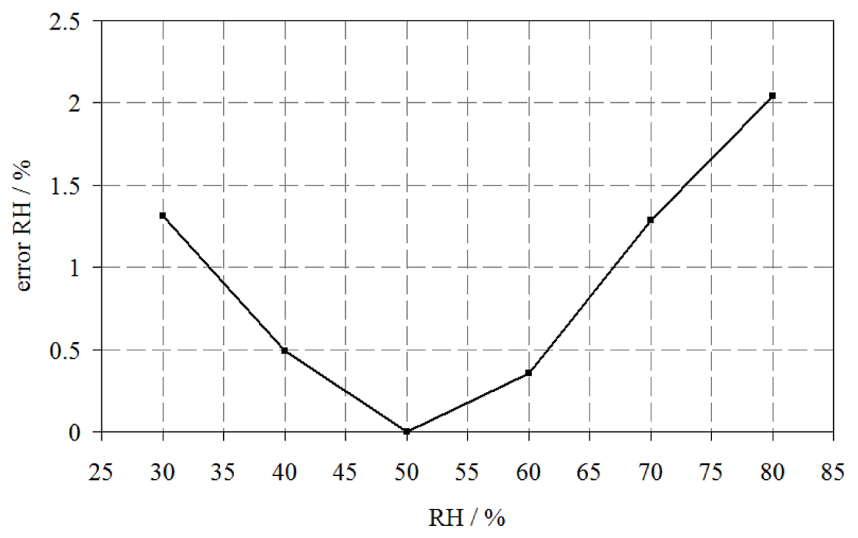

Figure 10. Error of the relative humidity using a one-point calibration at $50 \% \mathrm{RH}$ and $25^{\circ} \mathrm{C}$. The maximum error of four sensors is $\pm 2.2 \% \mathrm{RH}$.

\subsection{Calibration of the humidity sensor}

In this section, different calibration procedures for the humidity sensor are proposed. In Sect. 4.3.1 it was shown that a quadratic function is necessary to describe the output voltage as a function of the humidity. The cross-sensitivity to temperature is also non-linear. A straightforward way to compensate for the non-linear sensor response is a calibration at three different temperatures and three different relative humidities. A total of nine calibration points is expensive, but will result in an accuracy much better than $3 \%$ FS. If an accuracy of around $3 \% \mathrm{FS}$ is required, a two-point calibration is sufficient. The cheapest but less exact solution is a onepoint calibration. Here the accuracy will be around $5 \%$ FS.

For a better comparison to the values in datasheets of other sensors, the accuracy is specified in $( \pm \% \mathrm{RH})$ absolute instead of (\% FS) in the following (e.g. $1 \%$ FS in the range of $30-80 \% \mathrm{RH}$ is absolute $\pm 0.25 \% \mathrm{RH})$. 


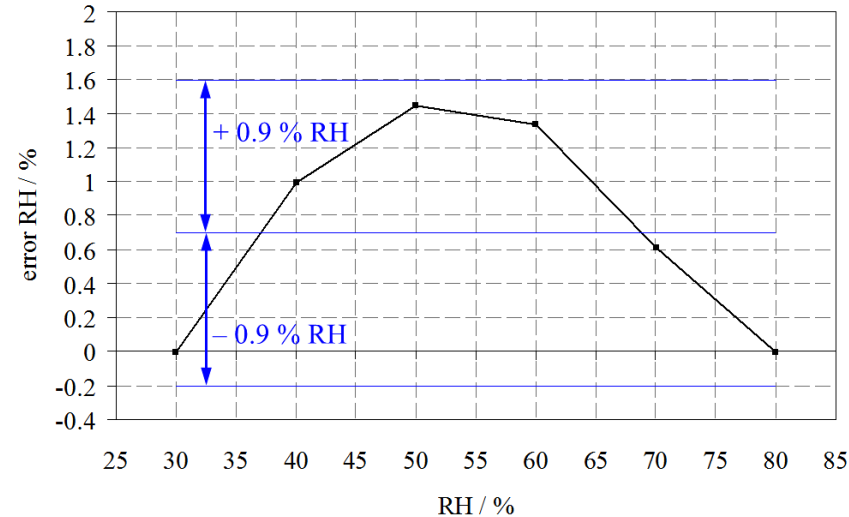

Figure 11. Error of the relative humidity using a two-point calibration at $30 \% \mathrm{RH} / 25^{\circ} \mathrm{C}$ and $80 \% \mathrm{RH} / 25^{\circ} \mathrm{C}$. To get a high absolute accuracy over the whole range, a typical offset of $0.7 \% \mathrm{RH}$ needs to be subtracted from the original linear interpolation data. The maximum error of the four sensors is then $\pm 0.9 \% \mathrm{RH}$.

\subsubsection{Calibration procedures}

In Sect. 4.3.1 a sensitivity of $(0.24-0.26) \mathrm{mV} / \% \mathrm{RH}$ was identified for the four sensors. The mean value of the sensitivity is $0.25 \mathrm{mV} / \% \mathrm{RH}$. With this value as a constant and an offset obtained from a single calibration point at $50 \% \mathrm{RH}$ and $25^{\circ} \mathrm{C}$, the measured humidity can be calculated by

$\mathrm{RH}\left(U_{\text {out }}\right)=U_{\text {out }} /$ Sens + Off.

Figure 10 shows the error of the relative humidity of one typical sensor at one-point calibration. For the four sensors investigated in this work, a maximum error of $\pm 2.2 \% \mathrm{RH}$ in the range of $30-80 \% \mathrm{RH}$ is obtained. A typical tolerance of $\pm 3 \% \mathrm{RH}$ between 20 and $80 \% \mathrm{RH}$ is given in the datasheet of the SHT20 humidity sensor. Also, the SHTC1 sensor from Sensirion, which is used in mobile phones, has the same tolerance. Therefore, a one-point calibration for humidity compensation of the new sensor is sufficient for many applications.

The best humidity sensor, SHT25 from Sensirion, has a typical tolerance of $\pm 1.8 \% \mathrm{RH}$ in a larger humidity range between 10 and $90 \%$ RH. All other values like hysteresis, repeatability and so on remained unchanged compared to the SHT20 sensor. This indicates that the only difference might be a calibration procedure with more calibration points.

To improve the accuracy of our humidity sensor, a twopoint calibration at $30 \% \mathrm{RH} / 25^{\circ} \mathrm{C}$ and $80 \% \mathrm{RH} / 25^{\circ} \mathrm{C}$ was investigated. The offset and the sensitivity of each sensor can be determined with a two-point calibration. Figure 11 shows the error of the relative humidity of one sensor for a twopoint calibration. To get a high absolute accuracy over the whole range, a typical offset of $0.7 \% \mathrm{RH}$ needs to be subtracted from the original linear interpolation data. The maximum error for all four sensors was then $\pm 0.9 \% \mathrm{RH}$, and

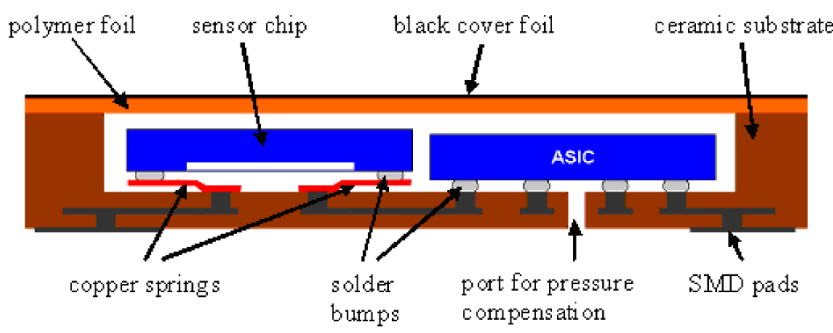

Figure 12. Novel flip-chip packaging technology for pressure and humidity sensor chips. High-temperature cofired ceramic (HTCC) is used for the package. The sensor chip is flip chip bonded on copper springs to obtain a stress-free package.

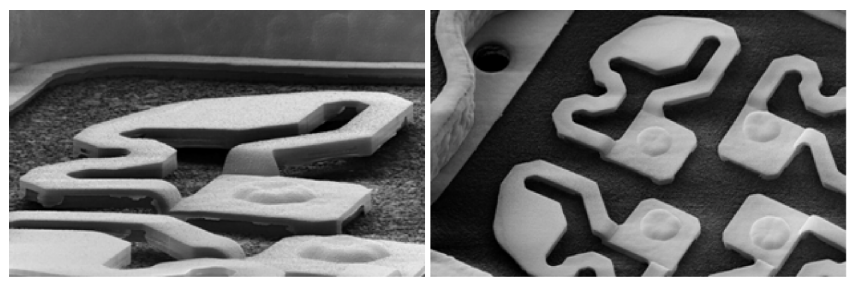

Figure 13. Copper springs in the cavity of the package. The springs are structured by photolithography. On the copper springs the sensor chip is flip-chip bonded.

therefore better than the SHT25 humidity sensor. However, the range between 30 and $80 \% \mathrm{RH}$ is smaller.

Temperature compensation in addition to humidity compensation is necessary. Two more additional points at different temperature therefore have to be measured. This means that, for temperature and humidity compensation, a fourpoint calibration at least should be used.

\subsection{Discussion of the results}

The measurements show that the new humidity sensor can be used to develop a commercial product. The accuracy will mainly depend on the effort spent on calibration. The measured sensitivity of $0.25 \mathrm{mV} / \% \mathrm{RH}$ is in quite acceptable agreement with the calculated value of $0.15 \mathrm{mV} / \% \mathrm{RH}$. By reducing the thickness of the polymer layer, the response time of the sensor could be decreased. This would also lead to a lower but still sufficient sensitivity.

\section{Outlook}

Piezoresistive pressure and humidity sensors are very sensitive to package stress. The applications in mobile devices call for a small package size. For barometric pressure sensors, wire bonding type packages, e.g. BMP180 from Bosch or LPS331AP from ST Microelectronics, and low stress flipchip packages, e.g., T5400 from EPCOS (Waber et al., 2013), have been developed that fulfill these requirements. Figure 12 shows the novel package with the copper springs and 
Fig. 13 a scanning electron microscope picture of the springs. This package is also suitable for the new humidity sensor. Shock and vibration tests with the new package and a sensor chip have been done. Guided free fall tests from a height of two meters result in a mean offset of around $0.1 \% \mathrm{RH}$. With the pressure sensor package from EPCOS, a footprint of $2.78 \times 2.23 \mathrm{~mm}^{2}$ and a height of $0.67 \mathrm{~mm}$ could be realized. ASICs, originally developed for barometric pressure sensors, could as well be used for the piezoresistive humidity sensor. Therefore, the development effort required to bring this new sensor onto the market is comparatively small. The sensor also offers the possibility of developing a combined pressure and humidity sensor. Most parts of the ASIC could be used for both measurements by adding a multiplexer between an analog-to-digital converter (ADC) and the sensor elements. With the integrated temperature sensor in the ASIC, we get an environmental sensor for mobile phones in one package. Furthermore, the development of an air density sensor can be interesting for hard disk manufacturers. Colin Johnson (Colin Johnson, 2009) wrote: "Without a measure of air density, hard drive heads can't run at minimum flight height without failing when barometric pressure changes dramatically, such as onboard an airborne plane."

The density of air $\rho$ depends on the pressure $p$, the humidity $\varphi$ and the temperature $T$. It applies Eq. (7):

$\rho(p, \varphi, T)=p \cdot \frac{\left(1-\varphi \cdot \frac{p_{\mathrm{svp}}}{p} \cdot\left(1-\frac{R_{\mathrm{a}}}{R_{\mathrm{wv}}}\right)\right)}{R_{\mathrm{a}} \cdot T}$,

with the gas constant of dry air $R_{\mathrm{a}}=287.058 \mathrm{~J} \mathrm{~kg}^{-1} \mathrm{~K}^{-1}$, the gas constant of water vapor $R_{\mathrm{wv}}=461.523 \mathrm{~J} \mathrm{~kg}^{-1} \mathrm{~K}^{-1}$, and the saturation vapor pressure $p_{\mathrm{svp}}$.

There are two possibilities of obtaining the air density. First, the humidity sensor chip described above together with a pressure sensor chip and an ASIC with a temperature sensor could be mounted into one package. With the multiplexer in the ASIC, the three signals of humidity, pressure and temperature could be alternately measured, and then the air density is calculated. A pressure and humidity sensor must be integrated into the package for this solution. The second possibility is to use a pressure sensor chip with a polymer pad on the membrane. For a certain size and thickness of the polymer pad, the bridge voltage will be proportional to the air density. The advantage of this solution would be the low cost and small size of the sensor.

\section{Conclusions}

A very small humidity sensor chip with a footprint of only $1 \times 1 \mathrm{~mm}^{2}$ based on the piezoresistive effect has been developed. The chip size was reduced by a factor of 10 compared to previous developments. Our experiments showed that the sensor can be used to measure the relative humidity of the air with a sensitivity of $0.25 \mathrm{mV} / \% \mathrm{RH}$.
Compared with humidity sensors applied in mobile phones today, the new sensor utilizing the current packaging technology of the EPCOS T5400 pressure sensor would be similar or smaller in size. Even in a first one-shot lab experiment, the performance of our piezoresistive sensor is at least comparable to current capacitive humidity sensors like the SHTC1 from Sensirion. With further optimization we see a good potential to meet whatever requirement is most important for the target application: either excellent accuracy or low costs by simple one-point calibration, or quick response times at still sufficient sensitivity by reducing the polymer thickness.

Edited by: U. Schmid

Reviewed by: two anonymous referees

\section{References}

Bosch: Sustainability in a Connected World is Bosch focus, International CES, Bosch press release, 2014.

Buchhold, R., Nakladal, A., Gerlach, G., Sahre, K., Müller, M., Eichhorn, K.-J., Herold, M., and Gauglitz, G.: A study on the microphysical mechanisms of adsorption in polyimide layers for microelectronic applications, J. Electrochem. Soc., 145, 40124018, 1998a.

Buchhold, R., Nakladal, A., Gerlach, G., Sahre, K., Eichhorn, K.J., Herold, M., and Gauglitz, G.: Influence of moisture-uptake on mechanical properties of polymers used in microelectronics, MRS Proceedings, 511, 359-364, 1998 b.

Buchhold, R., Nakladal, A., Gerlach, G., Sahre, K., Eichhorn, K.J., and Müller, M.: Reduction of mechanical stress in micromachined components caused by humidity-induced volume expanison of polymer layers, Microsystem Technol., 5, 3-12, 1998c.

Buchhold, R., Nakladal, A., Gerlach, G., and Neumann, P.: Design studies on pieoresistive humidity sensors, Sensors Actuators B, 53, 1-7, 1998d.

Buchhold, R., Nakladal, A., Gerlach, G., Herold, M., Gauglitz, G., Sahre, K., and Eichhorn, K.-J.: Swelling behavior of thin anisotropic polymer layers, Thin Solid Films, 350, 178-185, 1999.

Colin Johnson, R.: MEMS barometers boost hard drives, GPS, Electronic Engineering Times, 2009.

Gerlach, G. and Sager, K.: A piezoresistive humidity sensor, Sensors Actuators A, 43, 181-184, 1994.

Guenther, M., Sahre, K., Suchaneck, G., Gerlach, G., and Eichhorn, K.-J.: Influence of ion-beam induced chemical and structural modification in polymers on moisture uptake, Surface Coating. Technol., 142-144, 482-488, 2001.

Lane, N. D., Miluzzo, E., Lu, H., Peebles, D., Choudhury, T., and Campbell, A. T.: A survey of mobile phone sensing, IEEE Communications Magazine, 48-9, 140-150, 2010.

Lee, C.-Y. and Lee, G. B.: Humidity sensors: a review, Sensor Letters, 3, 1-15, 2005.

Mayer, F. and Lechner, M.: Sensirion brings temperature and humidity sensors to the mobile phone, MEMS Trends, 15, 12-13, 2013.

Sager, K., Gerlach, G., and Schroth, A.: A humidity sensor of a new type, Sensors and Actuators B, 18-19, 85-88, 1994. 
Sone, H., Fujinuma, Y., Hieida, T., Chiyoma, T., Okano, H., and Hosaka, S.: Picogram mass sensor using microcantilever, Proc of SICE Annual Conf., pp. 1508-1513, 2004a.

Sone, H., Fujinuma, Y., Hieida, T., Chiyoma, T., Okano, H., and Hosaka, S.: (Picogram mass sensor using microcantilever), Jap. J. Appl. Phys., 43, 4663-4666, 2004b.

Waber, T., Pahl, W., Schmidt, M., Feiertag, G., Stufler, S., Dudek, R., and Leidl, A.: Flip-chip packaging of piezoresistive barometric pressure sensors, Proc. SPIE, 8763, 876321, doi:10.1117/12.2016459, 2013.

Waber, T., Pahl, W., Schmidt, M., Feiertag, G., Stufler, S., Dudek, R., and Leidl, A.: Temperature characterization of flip-chip packaged piezoresistive barometric pressure sensors, Microsystem Technologies, 20, 861-867, 2014.
Wagner, T., Krotzky, S., Weiß, A., Sauerwald, T., Kohl, C.-D., Roggenbuck, J., and Tiemann, M.: A high temperature capacitive humidity sensor based on mesoporous silica, Sensors, 11-3, 3135-3144, 2011.

Wasisto, H. S., Merzsch, S., Huang, K., Stranz, A., Waag, A., and Peiner, E.: Simulation and characterization of silicon nanopillar-based nanoparticle sensors, Proc. SPIE, 8763, 87632D, doi:10.1117/12.2016970, 2013.

Yamazoe, N. and Shimizu, Y.: Humidity sensors: Principles and application, Sensors and Actuators, 10, 379-398, 1986. 